\title{
Alteración del pH salival en pacientes fumadores con enfermedad periodontal
}

\section{Salivary pH alterations in smoker patients with periodontal disease}

\author{
OSORIO GONZÁLEZ AY*, \\ BASCONES MARTÍNEZ A **, \\ VILLARROEL-DORREGO $\mathbf{M} * * *$
}

\begin{abstract}
Osorio González AY, Bascones Martínez A, Villarroel-Dorrego M. Alteración del $\mathrm{pH}$ salival en pacientes fumadores con enfermedad periodontal. Av Periodon Implantol. 2009; 21, 2: 71-75
\end{abstract}

\section{RESUMEN}

Introducción: El tabaquismo es un factor de riesgo muy importante en la enfermedad periodontal. Para que la nicotina sea absorbida efectivamente el $\mathrm{pH}$ de la saliva pareciera sufrir cambios en sus propiedades. Se estima que el $\mathrm{pH}$ en la saliva no estimulada oscila entre 5,5 a 7,9 , aumentando a medida que aumenta el flujo salival. Alteraciones del $\mathrm{pH}$ pudieran ser una de las causa de la activación de la enfermedad periodontal en fumadores.

Objetivo: El propósito de este trabajo fue determinar la asociación entre el pH salival y la enfermedad periodontal en pacientes fumadores.

Materiales y métodos: 20 individuos fueron incluidos en el estudio posterior a evaluación periodontal. 10 conformaron el grupo de fumadores y 10 no fumadores. La saliva no estimulada de cada individuo fue recolectada y el $\mathrm{pH}$ determinado. Las medias de $\mathrm{pH}$ se compararon estadísticamente usando el software SPSS 11.5.

Resultados y conclusiones: La diferencia entre el $\mathrm{pH}$ de ambos grupos no fue estadísticamente significativa, sin embargo si se observaron diferencias en relación al estatus periodontal de cada uno. Pareciera que el pH salival pudiera contribuir en la enfermedad periodontal de pacientes fumadores sin embargo no el factor fundamental en dicha patogénesis.

PALABRAS CLAVE: Tabaquismo, enfermedad periodontal, saliva.

\section{SUMIMARY}

Introduction: Several studies have found smoking a significant risk factor for periodontitis. Smoking alters salivary $\mathrm{pH}$ in order to improve nicotine absorption. Resting salivary $\mathrm{pH}$ estimate a range of 5.5 to 7.9 , with the higher $\mathrm{pH}$ exhibited upon increased salivary flow. Imbalance of $\mathrm{pH}$ might constitute a local factor for periodontal disease activation.

Objective: The purpose of this study was to determine the association of salivary $\mathrm{pH}$ with periodontal disease status in smokers and non-smokers.

Materials and methods: 20 individuals were included in the study. 10 smokers and 10 non-smoker. Periodontal status was assed and the whole mouth unstimulated saliva was collected, measured and $\mathrm{pH}$ estimated extra orally. Means of $\mathrm{pH}$ were compared statically using SPSS 11.5.

Results and conclusions: The difference between mean $\mathrm{pH}$ of smokers and non-smokers was statistically significant. Different levels of periodontal disease were found on both groups. The results

*, *** Maestría Medicina Bucal. Universidad Central de Venezuela.

* Universidad Complutense de Madrid. 
of this study suggest that $\mathrm{pH}$ alteration may contribute to periodontal disease in smoker patients however it is not the main aspect of the pathogenesis.

KEY WORDS: Smoking, periodontal disease, salivary.

Fecha de recepción: 18 de octubre de 2008.

Fecha de aceptación: 23 de octubre de 2008.

\section{INTRODUCCIÓN}

El tabaquismo es definido por la Organización Mundial de la Salud como una enfermedad crónica que produce dependencia y es, desde hace ya algunos años, la primera causa de muerte no natural en los países desarrollados (1).

Estudios epidemiológicos han demostrado que el tabaquismo es un factor de riesgo significativo para el desarrollo de enfermedades periodontales y la severidad de la enfermedad aumenta con la frecuencia del uso del tabaco o cigarrillo (2-5).

Las teorías que explican como el tabaquismo induce enfermedad periodontal están fundamentadas en una alteración en el equilibrio huésped-bacteria, con una mayor colonización de patógenos periodontales por la disminución de la respuesta inmunitaria, lo cual conduce a un incremento en la gravedad y extensión de la enfermedad periodontal. Además ocurre una disminución del fluido crevicular y de la concentración oxígeno en los tejidos lo cual se traduce en un aumento del factor de necrosis tumoral alfa y de la prostaglandina E2, importantes mediadores de la reabsorción ósea (6-8).

Por otra parte, reportes de la literatura han sugerido una asociación entre el tabaquismo y el funcionamiento de las glándulas salivales. Estudios preliminares en saliva estimulada de fumadores y no fumadores sugieren una baja capacidad amortiguadora en fumadores (9); y otros estudios subrayan una correlación negativa entre la baja capacidad amortiguadora de pacientes fumadores y altos valores de $\mathrm{pH}(10)$.

El primer lugar que toca el humo del tabaco es la boca y luego el pulmón que es donde se realiza la absorción a todo el organismo. Ésta se hace con extraordinaria rapidez y por la circulación llega al corazón, des- de donde es impulsada llegando en pocos segundos al cerebro donde ejerce su acción sobre el sistema nervioso (11). La nicotina es una sustancia que se absorbe fundamentalmente a través de los pulmones (de un 60 a un $90 \%$ ) y en menor medida a través de la mucosa bucal y plexos sublinguales (4 a 40\%) y de la piel, siendo en este caso la absorción variable y dependiente de varios factores, como son la temperatura y el $\mathrm{pH}$, el grado de humedad e higiene personal, entre otros.

La mayoría de la nicotina está ligada químicamente a las sustancias "ácidas" del humo de tabaco, por lo que es no volátil y es absorbida lentamente por el fumador. Conforme aumenta el pH una mayor proporción de nicotina de las sustancias "ácidas" se libera y se convierte en nicotina libre, la cual es volátil y es absorbida más rápidamente por la persona. Estos cambios en el $\mathrm{pH}$ pudieran ser la causa de las alteraciones de la mucosa bucal.

El objetivo del presente trabajo fue determinar la asociación entre el pH salival y la enfermedad periodontal en pacientes fumadores y no fumadores

\section{MATERIALES Y MÉTODOS}

Se estudió un grupo de 20 pacientes: 10 fumadores y 10 no fumadores con enfermedad periodontal, que acudieron a la Facultad de Odontología de la Universidad Central de Venezuela.

\section{Criterios de inclusión}

Pacientes diagnosticados con tabaquismo los cuales:

- Consuman al menos 10 cigarrillos diarios.

- Tabaquismo por un tiempo mínimo de 10 años.

- Pacientes con enfermedad periodontal activa. 


\section{Criterios de exclusión}

- Pacientes con enfermedades sistémicas que afecten la estructura de las glándulas salivales.

- Pacientes con alteraciones vasculares o neurológicas que afecten la función de las glándulas salivales.

- Pacientes que reciban tratamiento farmacológico que afecte la tasa de flujo salival.

- Pacientes que estén recibiendo tratamiento de radioterapia de cabeza y cuello y/o quimioterapia.

- Pacientes sin enfermedad periodontal.

Se tomaron 3 muestras de saliva a los pacientes fumadores y no fumadores, recolectadas en 2 sesiones en días separados en un rango de horario comprendido entre 9:00 am y 11:00 am para evitar alteraciones indeseadas por efecto del ciclo circadiano (12). Para el día de la recolección se indicó a los participantes no ingerir alimentos, ni bebidas 1 hora antes de la toma de la muestra. A los pacientes fumadores se les instó a fumar 1 cigarrillo previa toma de la muestra.

La muestra de saliva obtenida se depositó en un tubo de ensayo milimetrado. Posteriormente se transfirió a un microtitulador para la medición de $\mathrm{pH}$. Se estableció el $\mathrm{pH}$ en forma directa colocando dentro de la muestra de saliva un electrodo de $\mathrm{pH}$ Accumet Cientific conectado a un potenciómetro marca Orion Research, modelo 710 A previamente calibrado.

Las medias de $\mathrm{pH}$ fueron comparadas mediante el test $t$ de Student y $\mathrm{p}<0,05$ fue considerado estadísticamente significativo.

\section{RESULTADOS}

El grupo de pacientes no fumadores estuvo conformado por 6 mujeres y 4 hombres con una media de edad de 49 años. El grupo de fumadores estuvo constituido por 9 mujeres y 1 hombre con una media de edad de 40 años. El diagnóstico periodontal de los pacientes no fumadores correspondió mayormente a gingivitis crónica (8 pacientes) (Fig. 1) y sólo 2 casos se diagnosticaron como periodontitis crónica. En cambio los pacientes fumadores mostraron lesiones periodontales más severas ( 3 casos de gingivitis crónica y 7 casos de periodontitis crónica) (Fig. 2).

En relación al pH salival la media del grupo no fumador fue de 6,578 $\pm 0,1402$. Hubo un incremento estadísticamente significativo del $\mathrm{pH}$ en el grupo fumador

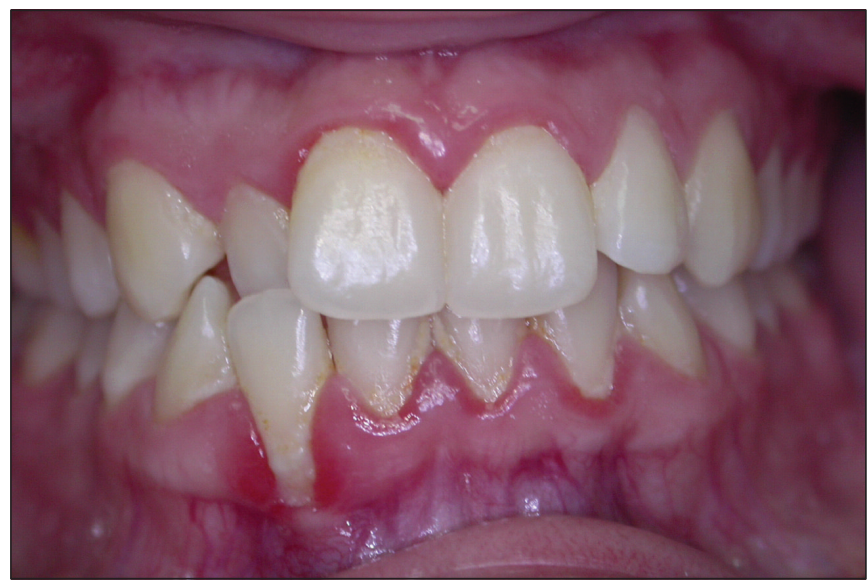

Fig. 1: Paciente no fumador con diagnóstico de gingivitis crónica.

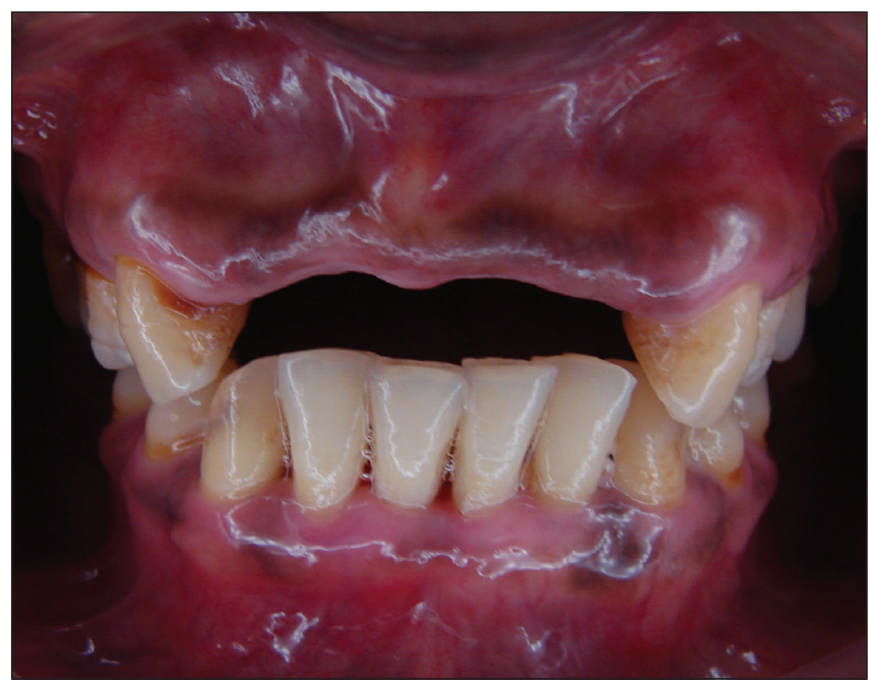

Fig. 2: Paciente fumador con diagnóstico de periodontitis crónica.

$(p=0,01)$, conformando una media de $7,367 \pm 0,1306$ (Figs. 3 y 4 ).

\section{DISCUSIÓN}

No hay duda que el tabaquismo es un factor que aumenta el riesgo de enfermedad periodontal $(3,4)$ o un agente importante que empeora el pronóstico de esta enfermedad $(3,7,13)$.

Los fumadores acumulan marcadamente más cálculo dental que los no fumadores, y la cantidad de cálculo está correlacionado con la frecuencia del tabaquismo 


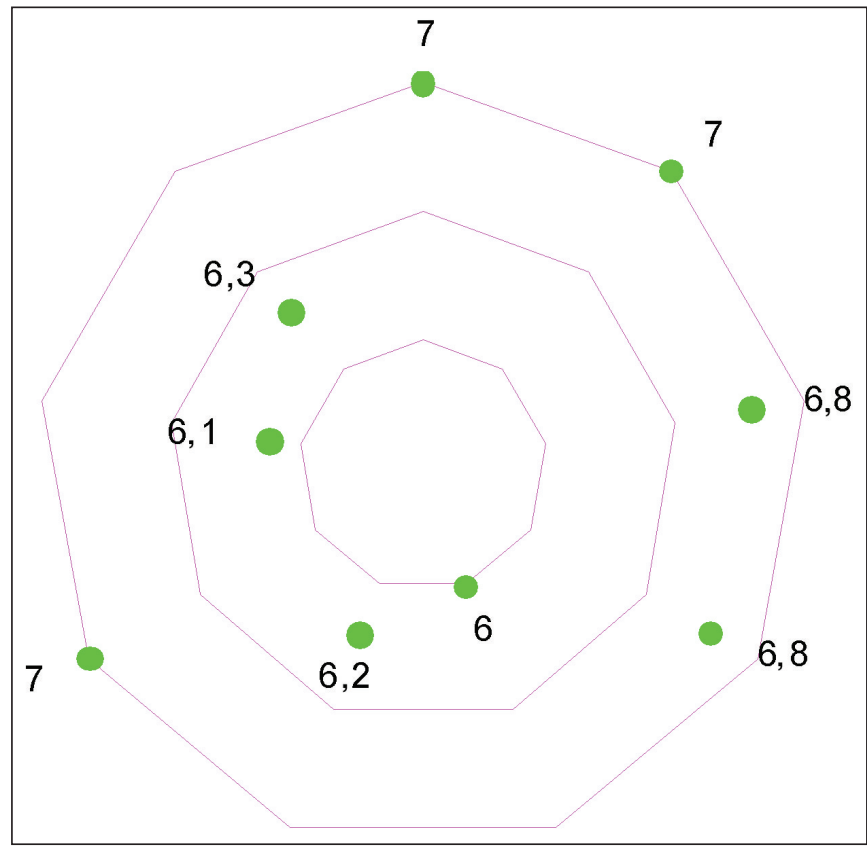

Fig. 3: Valores de $\mathrm{pH}$ del grupo de pacientes no fumadores.

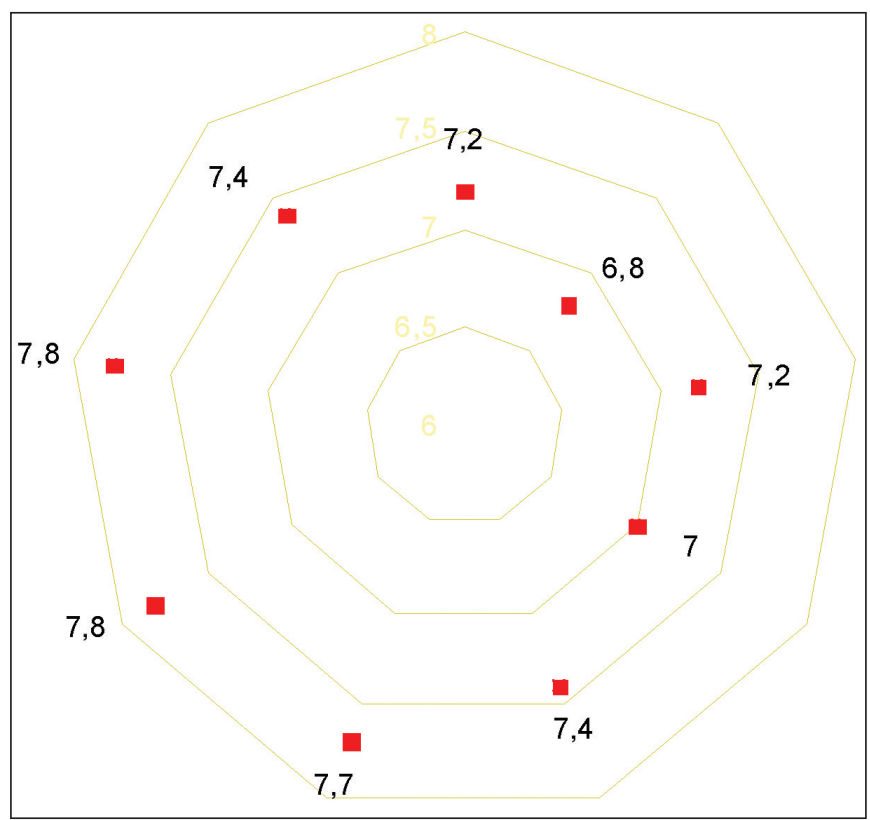

Fig. 4: Valores de pH del grupo de pacientes fumadores.

$(4,13)$. El tabaquismo también está asociado con un aumento en el riesgo de pérdida de la adhesión periodontal y la formación de sacos periodontales, así como también la pérdida ósea. Los efectos adversos del ci- garrillo sobre el periodonto se correlacionan bien con la cantidad del consumo diario y su duración $(14,15)$.

El pH salival mostró un incremento en los pacientes fumadores. Estos cambios en la alcalinidad salival benefician la absorción de la nicotina y pudieran ser la causa de mayor acumulo de placa y cálculo y por ende de enfermedad periodontal. Contrariamente se han reportado valores significativamente disminuidos de $\mathrm{pH}$ en fumadores con respecto al $\mathrm{pH}$ de los no fumadores (10). Esta disminución del $\mathrm{pH}$ fue asociada con otros factores que resultaron en el incremento de la concentración del ión de hidrógeno y la activación del sistema nervioso autónomo sobre la glándula salival (10).

Mayormente se cree que el uso del tabaco en un largo período de tiempo deprime o inactiva los receptores del gusto y el reflejo salival. Presumiblemente, esto pueda llevar a unos receptores del gusto alterados y a cambios en la secreción salival (16).

La evidencia de estudios seccionales y casos control en varias poblaciones demuestra que los adultos fumadores tienen aproximadamente 3 veces más probabilidades de padecer periodontitis que los no fumadores. Así mismo, la asociación entre fumar y pérdida de inserción está fuertemente relacionada con la definición de periodontitis, que establece que ésta es más severa en pacientes fumadores, encontrándose que la pérdida de inserción en fumadores es 6 veces mayor que en los no fumadores $(17,18)$.

Aunque la enfermedad periodontal es una entidad multifactorial, el pH salival debe ser considerado como uno de los factores que contribuye a la iniciación o el desarrollo de las lesiones periodontales en los pacientes fumadores, quizás mediante la promoción de la formación de cálculo dental o el incremento de la absorción de nicotina, o seguramente ambos.

\section{BIBLIOGRAFÍA}

1. World Health Organization (WHO) Tobacco: deadly in any form of disguise. World non tobacco day 2006, Geneva, Switzeland WHO 2006.

2. Haber J, Wattles J, Crowley M, Mandell R, Joshipura K, Kent RL. Evidence for cigarette smoking as a major risk factor for periodontitis. J Periodontol. 1993;64(1): 16-23.

3. Amarasena N, Ekanayaka AN, Herath L, Miyazaki H. Tobacco use and oral hygiene as risk indicators for 
periodontitis.Community Dent Oral Epidemiol. 2002;30 (2):115-23.

4. Natto S, Baljoon M, Bergström J. Tobacco smoking and periodontal health in a Saudi Arabian population. J Periodontol. 2005;76(11):1919-26.

5. Haber J, Kent RL. Cigarette smoking in a periodontal practice. J Periodontol. 1992; 63(2):100-6.

6. Boström L, Linder LE, Bergström J. Smoking and cervicular fluid levels of IL-6 and TNF-alpha in periodontal disease. J Clin Periodontol. 1999;26(6):352-7.

7. Haffajee AD, Socransky SS. Relationship of cigarette smoking to attachment level profiles.J Clin Periodontol. 2001;28(4):283-95.

8. Krall EA, Dawson-Hughes B, Garvey AJ, García RI. Smoking, smoking cessation and tooth loss. J Dent Res 1997; 76(10):1653-9.

9. Heintze U. Secretion rate, buffer and number of lactobacilli and Streptococcus mutans of whole saliva of cigarette smokers and non-smokers. Scand J Dent Res 1984:92:294-301.

10. Parvinen, T. Stimulated salivary flor rate, $\mathrm{pH}$ and lactobacillus and yeast concentrations in non-smokers and smokers. Scand J Dent Res 1984; 92:315-8.

11. Warnakulasuriya S, Sutherland G, Scully C. Tobacco, oral cancer, and treatment of dependence.Oral Oncol. 2005;41(3):244-60.

12. Dawes C. The unstimulated salivary flow rate alter prolongad gum chewing. Arch Oral Biol 2005; 50: 561-3.
13. Muller HP, Stadermann S, Heinicke A. Longitudinal association between plaque and gingival bleeding in smokers and non-smokers. J Clin Periodontol. 2002; 29: 287-94.

14. Martínez-Canut P, Lorca A, Magan R. Smoking and periodontal disease severity. J Clin Periodontol. 1995; 22: 743-9.

15. Machuca G, Rosales I, Lacalle JR, Machuca C, Bullon P. Effect of cigarette smoking on periodontal status of healthy young adults. J Periodontol. 2000;71:738.

16. Khan GJ, Mehmood R, Salah-ud-Din, Ihtesham-ul-Haq. Effects of long-term use of tobacco on taste receptors and salivary secretion.J Ayub Med Coll Abbottabad. 2003; 15(4):37-9.

17. Johnson GK; Hill M. Cigarette smoking and the periodontal patient. J Periodontol. 2004 Feb;75(2):196209.

18. Johnson GK; Slach NA. Impact of Tobacco use on periodontal status. J Dent Educ. 2001 Apr;65(4):313-21.

\section{CORRESPONDENCIA}

\section{Mariana Villarroel Dorrego}

Centro de Especialidades Odontológicas

Departamento de Medicina Bucal.

Ave. Principal de Chuao.

Caracas-Venezuela

mvillarroeldorrego@gmail.com

mvillarroeldorrego@cantv.net 